\title{
ORIGINAL
}

\section{INGRESOS HOSPITALARIOS POR ENFERMEDADES INFECCIOSAS: INCIDENCIA DESDE 1999 HASTA 2003 EN UN AREA SANITARIA DE LA COMUNIDAD VALENCIANA}

\author{
Antonio Guerrero Espejo y Sofía Tomás Dols \\ Unidad de Investigación en Patología Infecciosa. Hospital de La Ribera. Alzira. Valencia.
}

\section{RESUMEN}

Fundamento: La enfermedad infecciosa persiste en la actualidad como una de las principales causas de mortalidad y morbilidad. Su naturaleza dinámica justifica el estudio epidemiológico de las mismas. El objetivo del trabajo fue analizar la incidencia de enfermedades infecciosas que con mayor frecuencia condicionan el ingreso hospitalario.

Métodos: Se realizó un estudio de las personas ingresadas entre los años 1999-2003 cuyo diagnóstico principal al alta hospitalaria se hubiera codificado, de acuerdo a la Clasificación Internacional de Enfermedades (CIE 9-MC), como enfermedad infecciosa en el Conjunto Mínimo Básico de Datos. Se seleccionaron 2.010 códigos de enfermedades infecciosas en actividad y se concentraron en 25 grupos adaptados al CIE 9MC. La población diana fue la correspondiente a un area de la Comunidad Valenciana.

Resultados: El 9,7\% de los ingresos durante el periodo estudiado (8.585 registros) se debió a una enfermedad infecciosa. La media de edad de las personas afectadas fue 38 años, la mediana 37, la desviación típica 31 y el rango entre 0-102 años. Predominó el ingreso de varones $(54,5 \%)$ sobre el de mujeres $(45,5 \%)$. La tasa de incidencia de ingresos por enfermedades infecciosas fue de 728 casos/100.000 habitantes y año. La mayor tasa de ingreso se produjo en niños y ancianos. Los grupos de enfermedades con mayor número de ingresos fueron, en orden decreciente, las enfermedades infecciosas digestivas, respiratorias y genitourinarias.

Conclusión: Los ingresos hospitalarios por enfermedades infecciosas alcanzaron la décima parte de los ingresos y 7 de cada 1.000 habitantes al año requirió ser hospitalizado con motivo de una patología infecciosa. Fue mayor la incidencia de las enfermedades intestinales, de aparato digestivo, respiratorio y genitourinario en la población infantil pero también, aunque en menor proporción, en los mayores de 65 años.

Palabras claves: Enfermedades infecciosas. Ingresos hospitalarios. Incidencia

\section{Correspondencia:}

Antonio Guerrero Espejo.

Área de Diagnóstico Biológico, Hospital de La Ribera.

Ctra. Alcira-Corbera Km. 1, 46600

Alcira (Valencia), España.

Correo electrónico: aguerrero@hospital-ribera.com

\section{ABSTRACT}

\section{Hospital Admissions for Infectious \\ Diseases: 1999-2003 Incidence in one Health District in the Autnomous Community of Valencia, Spain}

BacKground:. Infectious diseases are still currently one of the leading causes of death and illness. Their dynamic nature justifies the epidemiological study thereof. This study is aimed at analyzing the incidence of infectious diseases most often conditioning hospital admissions.

Methods: A study was conducted of those individuals admitted to hospital during the 1999-2003 period whose main diagnosis at admission to hospital had been encoded according to the International Disease Classification (ICD 9-CM) as an infectious disease in the Minimum Basic Data Set. A total of 2,010 active infectious disease codes were selected and were grouped into 25 groups as per the ICD 9-CM. The target population was that of one district in the Autonomous Community of Valencia.

Results: A total of $9.7 \%$ of the admissions during the period under study $(8,585$ records) were due to an infectious disease. Those affected averaged 38 years of age, median age of 37, standard deviation 31 and the range of 1-102 years. The admissions of males $(54.5 \%)$ prevailed over females $(48.5 \%)$. The incidence rate of admissions due to infectious diseases was 728 cases / 100,000 inhabitants / year. The highest rate of admissions was among young children and the elderly. The groups of diseases showing the largest number of admissions were, in descending order, infectious digestive, respiratory and genitourinary diseases.

Conclusion: The hospital admissions due to infections diseases totaled one tenth of the admissions, and 7/ 1,000 inhabitants / year required hospitalization for an infectious disorder. The highest incidence rates were found for intestinal diseases, digestive, respiratory and genitourinary tract diseases among the childhood-aged population, but also, although in smaller percentages, among individuals over 65 years of age.

Keywords: Communicable diseases. Incidence. Hospital. 


\section{INTRODUCCIÓN}

La enfermedad infecciosa persiste en la actualidad como una de las principales causas de mortalidad y morbilidad. Su naturaleza dinámica justifica el estudio epidemiológico de las mismas ${ }^{1,2}$. Si bien se han realizado diversos estudios epidemiológicos para conocer la incidencia real de la patología infecciosa, como causa de ingreso hospitalario, la mayor parte de los mismos, se han centrado en patologías concretas graves, como la neumonía, sin que se haya logrado conocer una estimación global de los ingresos hospitalarios o por algunos procesos infecciosos en países desarrollados $^{3-7}$.

El uso del conjunto mínimo de datos del hospital ha revelado su importancia como fuentes de información de la salud incluyendo el campo de enfermedades infecciosas $^{7}$. La información de las enfermedades infecciosas contenida en los registros de alta hospitalaria puede ser inestimables para la toma de decisión en la política sanitaria en países en vías de desarrollo con índices elevados de enfermedades infeccio$\operatorname{sas}^{4 ; 8}$, pero también en países desarrollados tiene utilidad ${ }^{9}$. El objetivo general ha sido conocer la incidencia de enfermedades infecciosas atendidas en el hospital en función del diagnostico principal de ingreso, y analizar los grupos de los distintos procesos infecciosos de forma comparativa entre niños, adultos y personas mayores.

\section{MATERIAL Y MÉTODOS}

\section{Diseño del estudio}

Se ha realizado un estudio descriptivo, observacional, retrospectivo, de población ingresada en el Hospital de la Ribera cuyo diagnostico principal fue una enfermedad infecciosa, mediante la revisión de 88.680 episodios de ingreso durante cinco años, desde enero de 1999 a diciembre del 2003, a través de los datos contenidos en el conjunto mínimo básico de datos al alta hospitalaria (CMBD) de los pacientes cuya fecha de alta hospitalaria fuese anterior al 1 de enero del 2004.

\section{Lugar del estudio}

El Departamento de Salud 11 de la Comunidad Valenciana está situado en el sur de la provincia de Valencia y la atención especializada la cubre el Hospital de La Ribera, desde el 1 de enero de 1999 con una media de camas de 262. Satisface la demanda de atención sanitaria generada en la comarca de La Ribera de la Comunidad Valenciana que abarca una población distribuida en más de 40 poblaciones. La institución cuenta con todos los servicios sanitarios especializadas salvo transplantes, quemados y cuidados intensivos de neonatología. El soporte físico de la historia clínica se encuentra en formato electrónico.

El Departamento de Salud 11 de la Comunidad Valenciana tuvo una población anual media asignada (por la Conselleria de Sanidad) de 236.230 habitantes $^{10}$ lo que representaba aproximadamente un 5,72\% de la población total de esta Comunidad. La densidad de población del área era de 181,14 - 272,74 habitantes/ $\mathrm{Km}^{2} \mathrm{El}$ 76,86 \% de la población vivía en municipios de más de 10.000 habitantes.

El porcentaje de hombres es inferior $(49,2 \%)$ al de mujeres $(50,8 \%)$ y predominaron las edades medias de la vida.

\section{Episodios de ingreso analizados}

Se han revisado un total de 88.680 episodios de ingresos hospitalarios analizando el ítem de diagnóstico principal contenidos en el CMBD de los pacientes dados de alta en el Hospital de la Ribera durante los años 1999, 2000, 2001,2002 у 2003 cuyo diag- 
nóstico principal a su alta fuese codificado como una enfermedad infecciosa en el CMBD.

El número de ingresos anuales han sido los siguientes. En los tres primeros años de funcionamiento del hospital, el número de ingreso ascendió: en 1999 ingresaron un total de 13.348 pacientes; en el 2000, 17.503 y en el 2001, 19204. Se siguió un ascenso progresivo más discreto del número de altas hospitalarias en los años sucesivos: en el 2002, 19.206 y en el 2003, 19.414 .

\section{Definición de caso}

Un ingreso hospitalario por enfermedad infecciosa se consideró a un paciente ingresado en el hospital de la Ribera con un diagnóstico principal de ingreso por enfermedad infecciosa de acuerdo con el juicio del médico que dio de alta al paciente.

Se seleccionó a cualquier paciente cuyo proceso nosológico fuera codificado de acuerdo con la CIE 9 MC, cuarta edición actualizada en enero del 2002 con alguno de los 2.010 códigos que hacen referencia a enfermedades infecciosas.

Se incluyeron todos los códigos del capítulo 1 de la CIE 9 MC que hace referencia a las enfermedades infecciosas salvo los efectos tardíos de enfermedades infecciosas y parasitarias (códigos 137139): enfermedades infecciosas intestinales (códigos del 001 al 009); tuberculosis (010-018); enfermedades bacterianas zoonósica (020-027); enfermedades bacterianas (030-041); infección del virus de la inmunodeficiencia humana (VIH) (042); poliomielitis y otras enfermedades virales del sistema nervioso central no transmitidas por artrópodos (045-49); enfermedades virales acompañadas de exantema (050-057) enfermedades virales portadas por artrópodos (060-066); otras enferme- dades debidas a virus y chlamydiae (070079); rickettsiosis y otras enfermedades portadas por artrópodos (080-088); sífilis y otras venéreas (090-099); otras enfermedades espiroquetales (100-104); micosis (110-118); helmintiasis (120-129) y, por último, otras enfermedades infecciosas y parasitarias (130-136).

Además, se incluyeron algunos códigos, que también hacen referencia a enfermedades infecciosas, seleccionados de los capítulos $6,7,8,9,10,11,12,13,15$, y 17 que respectivamente se refieren a las siguientes entidades: enfermedades del sistema nervioso central y órganos de los sentidos; enfermedades del aparato circulatorio; enfermedades del aparato respiratorio; enfermedades del aparato genitourinario; infeccion en gestación parto y puerperio; enfermedades de la piel y de los tejidos subcutáneos; enfermedades músculo-esquelético-conectivas; infecciones con origen en el periodo perinatal; infeccion por dispositivo, implante, injerto o posquirúrgica Se excluyeron algunos errores de codificación detectados al revisar la historia clínica y a pacientes que reingresaban en cortos espacios de tiempo por el mismo proceso (se eliminaron los ingresos sucesivos). Por tanto las fuentes de información fueron los datos establecidos en le CMBD complementados con los datos existente en la historia clínica informatizada de atención especializada (SIAS).

\section{Registro y análisis de las Variables}

La recogida de datos se hizo de forma automatizada empleando una aplicación informática a través de los registros citados. Posteriormente se depuraron los datos eliminando algunos errores de codificación.

Las variables principales fueron los diagnósticos de patología infecciosa como causa principal de ingreso detectados a través del sistema de codificación de la CIE-9 
MC y las sociodemográficas (edad, sexo y año de ingreso). Se estudio la presencia o ausencia de 2010 entidades nosológicas de la CIE-9-MC.

Los datos se archivaron en tablas de Excel donde se crearon campos para la identificación del paciente y episodio de ingreso, edad, sexo, lugar de residencia, fecha de ingreso, alta y codificación y código de la CIE -9MC, entre otros. Para el análisis de los datos, se agruparon las enfermedades infecciosas en 25 grupos.

\section{Análisis estadístico}

Se ha calculado la tasa de incidencia de ingresos por patología infecciosa estratificada por distintos tipos de enfermedades infecciosas y edades. Se consideró que la población de la Ribera atendida en el Hospital era una población dinámica estable de 236.230 habitantes; dicha cifra se obtuvo a partir de la población anual media asignada por la conselleria de sanidad teniendo en cuenta los datos del censo ${ }^{10}$. Se ha empleado una estadística descriptiva para la organización y resumen de los datos observados utilizando Excel y el paquete estadístico SPSS.

\section{RESULTADOS}

Se detectaron 8585 registros válidos de pacientes cuyo diagnóstico principal en el ingreso fue una enfermedad infecciosa entre los 88680 ingresos hospitalarios de los años 1999 a 2003. Por tanto, la tasa de ingresos por enfermedades infecciosas en relación con el número total de ingresos hospitalarios fue de 9,69\% siendo la tasa de incidencia de ingresos por enfermedades infecciosas de 727,60/100.000 habitantes y año.

La media de edad de estos pacientes fue de 38 años, la mediana de 37 y la desvia- ción típica de 31 (rango 0-102 años). La mayor frecuencia de ingresos se produjo en las edades extremas de la vida (niños y ancianos). Predominó el ingreso de varones con 4675 casos $(54,5 \%)$ sobre el de mujeres con 3910 casos $(45,5 \%)$.

El número de casos codificados por alguna enfermedad infecciosa, a lo largo de los años, ha ido aumentando progresivamente en relación al incremento progresivo de actividad de la institución y se mantuvo en todos los años el predominio del ingreso de hombres.

\section{Ingresos absolutos e incidencia por grupos de enfermedades}

Como puede observarse en la tabla $n^{\circ}$ 1 , los grupos de enfermedades que ocasionó un mayor número de ingresos por orden de frecuencia fueron las enfermedades del aparato respiratorio, del aparato genitourinario y del aparato digestivo (no intestinales). No obstante, el mayor número de ingresos correspondió a las enfermedades digestivas si se incluye la suma de los procesos codificados en el capítulo 9 de la CIE-9-MC (enfermedades de aparato digestivo) y las correspondientes a las enfermedades infecciosas intestinales (códigos 001-009) del capitulo 1. En la tabla, aparecen agrupadas diversos tipos de enfermedades infecciosas indicando la tasa de incidencia (de acuerdo con la población del área) y el numero absoluto de ingresos distribuidos según los grupos etarios.

Entre los diversos tipos de enfermedades infecciosas que dieron lugar a ingresos contabilizados en números absolutos a lo largo del periodo de estudio predominaron las enfermedades bacterianas, intestinales, digestivas, genitourinarias y respiratorias. Destacó que las enfermedades del grupo "enfermedades bacterianas" predominaron en los mayores de 64 años, las del grupo 
Tabla 1

Tasa de incidencia y número de ingresos absolutos en los 5 años por grupo de enfermedades

\begin{tabular}{|c|c|c|c|c|c|c|c|c|}
\hline \multirow{2}{*}{$\begin{array}{c}\text { Grupo de enfermedades } \\
\text { Rango de edad }\end{array}$} & \multicolumn{4}{|c|}{ Incidencia/100.000 habitantes y año } & \multicolumn{4}{|c|}{$N^{o}$ de ingresos en 5 años } \\
\hline & $0-14$ & $14-64$ & $>64$ & Total & $0-14$ & $14-64$ & $>64$ & Total \\
\hline Población & 35.321 & 162.48 & 4 & 42.947 & 240.75 & 2 & & \\
\hline Enf bacterianas & 28,3 & 13,4 & 227,3 & 53,7 & 50 & 109 & 488 & 647 \\
\hline Enf bacterianas zoonosicas & 1,1 & 0,6 & 1,9 & 0,9 & 2 & 5 & 4 & 11 \\
\hline Enf infecciosas intestinales & 384,5 & 14 & 48,4 & 74,5 & 679 & 114 & 104 & 897 \\
\hline Enf músculo-esquelético-conectivo & 7,4 & 7 & 14,4 & 8,4 & 13 & 57 & 31 & 101 \\
\hline Enf virales acompañadas de exantema & 26 & 2 & 3,3 & 5,7 & 46 & 16 & 7 & 69 \\
\hline Enf virales snc no transm. por artrópodos & 18,7 & 1,5 & 1,9 & 4,1 & 33 & 12 & 4 & 49 \\
\hline Enf virales por artrópodos & 0 & 0,1 & 0 & 0,1 & & 1 & & 1 \\
\hline Enf de la piel y de los tejidos subcutáneos & 20,4 & 13,4 & 32,6 & 17,9 & 36 & 109 & 70 & 215 \\
\hline Enf del ap. circulatorio & 0 & 6,9 & 9,3 & 6,3 & & 56 & 20 & 76 \\
\hline Enf del ap. digestivo & 165,9 & 109,5 & 115 & 118,8 & 293 & 890 & 247 & 1430 \\
\hline Enf del ap. genitourinario & 165,3 & 79,5 & 421,4 & 153,1 & 292 & 646 & 905 & 1843 \\
\hline Enf del ap. respiratorio & 689,7 & 44,2 & 221,7 & 170,5 & 1.218 & 359 & 476 & 2053 \\
\hline Enf sn y de los órganos de los sentidos & 44,2 & 10,5 & 14,9 & 16,2 & 78 & 85 & 32 & 195 \\
\hline Helmintiasis & 0,6 & 2,8 & 7,9 & 3,4 & 1 & 23 & 17 & 41 \\
\hline Infec asociadas al vih & 0,6 & 8,6 & 0,5 & 6 & 1 & 70 & 1 & 72 \\
\hline Infec con origen en el periodo perinatal & 39,1 & 0 & 0 & 5,7 & 69 & & & 69 \\
\hline Infec por dispositivo, implante o injerto & 1,1 & 10,3 & 65,2 & 18,8 & 2 & 84 & 140 & 226 \\
\hline Infec. en gestación parto y puerperio & 0 & 2,7 & 0 & 1,8 & & 22 & & 22 \\
\hline Micosis & 1,7 & 1 & 7,9 & 2,3 & 3 & 8 & 17 & 28 \\
\hline Otras enf virales y clamidias & 78,1 & 24,1 & 20 & 31,3 & 138 & 196 & 43 & 377 \\
\hline Otras espiroquetosis & 0 & 0,7 & 0,5 & 0,6 & & 6 & 1 & 7 \\
\hline Otras infec y parasitarias & 1,1 & 2,3 & 1,9 & 2,1 & 2 & 19 & 4 & 25 \\
\hline Rickettsiosis y otras enf por artrópodos & 0,6 & 0,5 & 0 & 0,4 & 1 & 4 & & 5 \\
\hline Sífilis y otras enfermedades venéreas & 0 & 0,2 & 0 & 0,2 & & 2 & & 2 \\
\hline Tuberculosis & 5,7 & 9,7 & 11,6 & 9,5 & 10 & 79 & 25 & 114 \\
\hline Total & 8.400 & $1.829,1$ & 6.138 & $3.561,8$ & 2.967 & 2.972 & 2.636 & 8.575 \\
\hline
\end{tabular}

"enfermedades infecciosas intestinales" en niños, las del grupo "enfermedades de la piel y tejido subcutáneo" y "genitourinario" en adultos, las del grupo de "infecciones respiratorias" en niños y la tuberculosis en adultos.

Al comparar la incidencia media de ingresos de niños, adultos y mayores por
100.000 habitantes y año en el área de la Ribera, se detectan notables diferencias entre algunos grupos y con respecto a la frecuencia relativa de ingresos. Fue mayor la incidencia de las enfermedades intestinales, de aparato digestivo, respiratorio y genitourinario en niños pero también, aunque en menor proporción, en mayores de 65 años. 


\section{Frecuencia de los diagnósticos de alta e incidencia de algunas enfermedades}

Ejemplo de la incidencia de alguna de las enfermedades más frecuentemente codificadas como causa principal de ingreso fueron las siguientes.

Para la apendicitis, sumando los datos de diversos códigos, fue 96/100.000 habitantes y año; predominó, como diagnóstico principal, la apendicitis sin mención de peritoni- tis seguida en segundo lugar de la apendicitis aguda con absceso peritoneal y en tercer lugar de la apendicitis aguda con peritonitis generalizada.

La incidencia de pielonefritis fue de 68/100.000 habitantes y año, excluyendo epígrafes sobre infecciones urinarias que no hacían referencia a infecciones urinarias altas. No obstante, las infecciones de vías urinarias no codificadas como pielonefritis se diagnosticaron con una similar frecuencia.

Tabla 2

Incidencia de los grupos de enfermedades por años de ingreso

\begin{tabular}{|c|c|c|c|c|c|c|}
\hline Nombre grupo & 1999 & 2000 & 2001 & 2002 & 2003 & $1999-2003$ \\
\hline Enf bacterianas & 36 & 39 & 55 & 67 & 72 & 54 \\
\hline Enf bacterianas zoonósica & 1 & $\mathbf{0}$ & $\mathbf{0}$ & 2 & 2 & 1 \\
\hline Enf infecciosas intestinales & 55 & 83 & 81 & 72 & 82 & 75 \\
\hline Enf músculo-esquelético-conectivo & 5 & 10 & 12 & 7 & 7 & 8 \\
\hline Enf virales acompañadas de exantema & 5 & 6 & 5 & 8 & 5 & 6 \\
\hline Enf virales del snc no transmitida por artrópodos & 3 & 4 & 3 & 5 & 6 & 4 \\
\hline Enf virales por artrópodos & $\mathbf{0}$ & $\mathbf{0}$ & $\mathbf{0}$ & $\mathbf{0}$ & $\mathbf{0}$ & 0 \\
\hline Enf de la piel y de los tejidos subcutáneos & 17 & 16 & 17 & 12 & 27 & 18 \\
\hline Enf del ap. circulatorio & 2 & 6 & 7 & 6 & 10 & 6 \\
\hline Enf del ap. digestivo & 121 & 125 & 116 & 111 & 121 & 119 \\
\hline Enf del ap. genitourinario & 113 & 133 & 172 & 161 & 187 & 153 \\
\hline Enf del ap. respiratorio & 133 & 158 & 166 & 193 & 203 & 171 \\
\hline Enf del sn y de los órganos de los sentidos & 15 & 16 & 13 & 16 & 20 & 16 \\
\hline Helmintiasis & 2 & 6 & 3 & 4 & 2 & 3 \\
\hline Infec asociadas al vih & $\mathbf{0}$ & $\mathbf{0}$ & 4 & 14 & 13 & 6 \\
\hline Infec con origen en el periodo perinatal & 3 & 7 & 5 & 6 & 7 & 6 \\
\hline Infec por dispositivo, implante o injerto & 5 & 18 & 17 & 26 & 29 & 19 \\
\hline Infec en gestación parto y puerperio & 1 & $\mathbf{0}$ & 2 & 4 & 2 & 2 \\
\hline Micosis & 2 & $\mathbf{0}$ & 3 & 3 & 3 & 2 \\
\hline Otras enf virales y clamidias & 17 & 29 & 34 & 33 & 43 & 31 \\
\hline Otras espiroquetosis & $\mathbf{0}$ & 1 & $\mathbf{0}$ & 1 & $\mathbf{0}$ & 1 \\
\hline Otras infec y parasitarias & $\mathbf{0}$ & 2 & 1 & 5 & 2 & 2 \\
\hline Rickettsiosis y otras enf por artrópodos & 1 & $\mathbf{0}$ & $\mathbf{0}$ & $\mathbf{0}$ & $\mathbf{0}$ & 0 \\
\hline Sífilis y otras enfermedades venéreas & $\mathbf{0}$ & 0 & 0 & 0 & $\mathbf{0}$ & 0 \\
\hline Tuberculosis & 12 & 6 & 10 & 9 & 10 & 10 \\
\hline Total general & 552 & 666 & 727 & 767 & 854 & 713 \\
\hline
\end{tabular}


Entre las infecciones respiratorias destacamos la bronquiolitis con una incidencia de 55/100.000 habitantes y año y la neumonía con una incidencia de 20/100.000 habitantes y año.

Entre las infecciones intestinales, mencionamos a la gastroenteritis por Salmonella y 23/100.000 habitantes y año y a la diarea por rotavirus con una incidencia de 9,48 por 100.000 habitantes y año.

La tasa de incidencia de las infecciones del sistema nervioso y órganos de los sentidos fue de 16,2 casos/100.000 habitantes y año, aunque entre los niños esa incidencia se elevó a 44,2 casos/100.000 habitantes y año.

La tasa de incidencia de ingresos por meningitis infecciosas fue de 8 casos/100.000 habitantes y año. El mayor número de ingresos correspondieron a las meningitis víricas no filiadas $(35,1 \%$ de los casos) y la meningitis neumocócica $(16 \%$ de los casos).

La tasa de ingresos por micosis fue de 2,3 por $100.000 /$ habitantes y año en la población general con un enorme predominio de las mismas en personas mayores donde alcanzó la tasa de 7,9 por $100.000 /$ habitantes y año.

Los siguientes grupos de enfermedades alcanzaron una incidencia inferior a 1 caso por 100.000 habitantes y año: enfermedades bacterianas zoonóticas, enfermedades virales transmitidas por artrópodos, rickettsiosis y otras enfermedades transmitidas por artrópodos, sífilis y otras enfermedades venéreas así como otras espiroquetosis.

\section{Análisis de frecuencias por año}

Al comparar las frecuencias correspondientes a los años 1999, 2000, 2001, 2002 y 2003 se observa un incremento de los casos pero no existieron diferencias significativas en cuanto a la edad, sexo, ni distribución de enfermedades por año, salvo el menor número de ingresos codificados como infecciones asociadas al VIH en los primeros años (tabla $n^{\circ} 2$ ).

\section{DISCUSIÓN}

Siete personas por cada 1000 habitantes y año requirieron un ingreso hospitalario por una enfermedad infecciosa. Esta tasa de ingresos por enfermedades infecciosas, representa la mitad de la detectada en 1994 en Estados Unidos donde la tasa de hospitalización para enfermedades infecciosas fue de 15,4 por 1000 habitantes $^{2}$. Entre las principales razones de la reducción de las enfermedades infecciosas en el mundo en el último siglo se encuentra la mejora de la atención sanitarias y de higiene, el uso masivo de vacunas efectivas y el uso de agentes antimicrobianos.

No obstante, y a pesar del nivel sanitario de España, una décima parte de los ingresos hospitalarios $(9,69 \%)$ se debió a una enfermedad infecciosa. Existen pocos datos en la literatura, para comparar estas tasas entre diversos países y además no se han empleado métodos idénticos en la obtención de $\operatorname{los}_{\operatorname{mismos}}^{9}(2 ; 9 ; 11)$. Así, Mills y colaboradores durante el periodo 19882000 , detectó un $12.6 \%$, aunque incluía también los efectos tardíos de enfermedades infecciosas o consecuencia del tratamiento o profilaxis de enfermedades infecciosas ${ }^{11}$.

El predominio de ingresos de varones $(54,5 \%)$ sobre el de mujeres $(45,5 \%)$ a pesar del mayor porcentaje de mujeres que de hombres en la población atendida sugiere un mayor riesgo del hombre a padecer una enfermedad infecciosa grave que requiera atención hospitalaria.

La mayor frecuencia de ingresos en números absolutos se produce en las edades 
extremas de la vida (niños y ancianos) pero es variable el número absoluto de ingresos según la edad en los distintos grupos de enfermedades. No obstante, al tener en cuenta la población del área, encontramos diferencias notables en las tasas de incidencia según la edad de los pacientes ingresados: en niños fue mayor la incidencia de las enfermedades intestinales, de aparato digestivo, respiratorio y genitourinario pero también, aunque en menor proporción, en los mayores de 65 años. Se deduce que los niños están sometidos a un mayor riesgo de padecer una enfermedad infecciosa que requiere ingreso que los otros grupos de población.

Las diarreas continúan siendo una causa frecuente de hospitalización en los países desarrollados, especialmente entre niños ${ }^{12}$. Incluso entre adultos, la diarrea puede representar un $1.5 \%$ de todas las hospitalizaciones ${ }^{13}$. Entre las enfermedades infecciosas intestinales en el area sanitaria estudiada destaca por su frecuencia la gastroenteritis por Salmonella, con una incidencia de 23 casos por 100.000 habitantes y año, y, entre las no bacterianas, la diarrea por rotavirus con una incidencia de 9,5 por 100000 habitantes y año. La importancia relativa de todas las causas de diarreas se ha analizado en otros países mediante un sistema similar al CMBD. Así, Nelson y col. en Hong Kong detectaron que el $11 \%$ de los niños de menos de 5 años dados de alta lo fueron con el diagnóstico primario de diarrea de las cuales el $74 \%$ fueron codificadas como no específicas, $10,4 \%$ como por rotavirus, el $11 \%$ como por Salmonella y el $5 \%$ como otras víricas o bacterianas ${ }^{14}$. Otros sistemas de información centrados en la microbiología o en una entidad concreta pueden ofrecer datos más ajustados en cuanto a la etiología. Así, un 14\% de los patógenos responsables de gastroenteritis corresponde a rotavirus en el sistema nacional de información microbiológica; Otros autores han estimado incidencias anuales de hospitalización por rotavirus entre 1-2,5 casos por 1000 niños de 5 años o menor edad (durante el invierno, 2.5 por $1000)^{15,16}$.

La apendicitis, seguida de la colecistitis, fue la infección intraabdominal predominante. La incidencia de apendicitis de 96 casos por 100.000 habitantes y año, mide la incidencia real de esta enfermedad ya que su diagnóstico condiciona el ingreso hospitalario. En USA la tasas de apendicitis como causa de ingreso hospitalario se aproxima al $0,6 \%$ de los ingresos ${ }^{17}$; nuestra tasa es superior, $1,3 \%$ de todos los ingresos, $\mathrm{y}$ un $13 \%$ de los ingresos por patología infecciosa. La proporción de casos de apendicitis con perforación es superior en personas mayores $^{18}$.

Causas frecuentes de ingreso por infección respiratoria son la neumonía o bronquiolitis. Se estima que la incidencia de la neumonía adquirida en la comunidad alcanza, una incidencia de 2-10 casos por 1.000 habitantes/año ${ }^{19-21}$. La incidencia como causa principal de ingreso, inferior a lo esperada, sugiere que se haya codificado algunos ingresos con neumonía bajo otros epígrafes. Además, aunque entre los patógenos más frecuentes de la neumonía adquirida en la comunidad están $S$. pneumoniae, H. influenzae, Chlamydia pneumoniae y Legionella pneumophi$l a^{22,23,24 ; 25,26}$, no predominaron estos patógenos pues en la codificación se especificó con mayor frecuencia, como diagnóstico principal, la etiología estafilocócica, por Pseudomonas y viral; posiblemente el CMBD al alta no permita un conocimiento adecuado de la distribución etiológica de las neumonías. La bronquiolitis es una de las principales causas de hospitalización en los niños con menos de un año. La incidencia de ingresos por bronquiolitis por el virus respiratorio sincitial (55 casos/100.000 habitantes y año en el área de la Ribera ) superó al de otros patógenos de acuerdo con otros estudios ${ }^{27}$. 
Una de las fuentes de información sanitaria de un país es el conjunto mínimo básico de datos al alta hospitalaria pero tiene limitaciones de cobertura si se considera solo el diagnóstico principal del alta hospitalaria. Pueden ser poco sensibles para determinados diagnósticos y un mismo diagnostico puede codificarse de diferente manera. Si se comparasen, con bases de datos microbiológicas podrían encontrarse deficiencias. En 1998, Madsen y colaboradores determinaron la calidad de los datos del registro del sepsis en una base de datos del alta del hospital, comparando datos del alta con datos de una base de datos automatizada del bacteriemia en un departamento regional de microbiología clínica; concluyeron que el registro del alta del hospital reveló clasificaciones equivocadas, y el sistema fue encontrado no conveniente para la vigilancia del bacteriemia ${ }^{28}$.

No obstante, este recurso posee un gran potencial aunque los datos basados en estudios de la causa principal de ingreso solo pueden dar lugar a una aproximación de la incidencia real de las enfermedades infecciosas en atención especializada ya que no van a tener en cuenta las infecciones nosocomiales producidas durante el ingreso ni los diagnósticos secundarios. Estas bases de datos excluyen también los diagnósticos de pacientes en atención primaria o consultas externas hospitalarias por procesos no graves. Prescinden además de los correspondientes a los pacientes atendidos en los servicios de urgencia y a los fallecimientos no ingresados en el hospital. Las tasas de ingresos por tuberculosis solo son un reflejo de la incidencia de la enfermedad. Así fue de 9,5 por 100.000/habitantes y año en la población general, se reduce en niños a $5,7 \%$ por $100.000 /$ habitantes y año y se eleva en las personas mayores a $11,6 \%$ por 100.000/habitantes y año, probablemente como expresión del envejecimiento del sistema inmunitario (primario o secundario a otras enfermedades) que facilita la reactivación de la enfermedad tuberculosa; no obs- tante nuestras cifras reales (incluyendo diagnósticos de consultas) son un cerca de un $50 \%$ superiores.

Sin embargo, el gran volumen de datos que proporciona, fácilmente disponibles, del ingreso hospitalario representa una fuente atractiva para la investigación epidemiológica. La información del conjunto mínimo de datos del hospital, cuando se utiliza correctamente, pueden ser una herramienta eficiente para la vigilancia, la identificación de la tendencia de la salud, y el estudio epidemiológico en enfermedades infecciosas aunque se debe complementar con otros registros entre los que destacan los microbiológicos.

\section{BIBLIOGRAFÍA}

1. Armstrong GL, Conn LA, Pinner RW. Trends in infectious disease mortality in the United States during the 20th century. JAMA 1999; 281:61-6.

2. Simonsen L, Conn LA, Pinner RW, Teutsch S. Trends in infectious disease hospitalizations in the United States, 1980-1994. Arch Intern Med 1998; 158:1923-8.

3. Almirall J, Bolíbar I, Vidal J, Sauca G, Coll P, Niklasson B, et al. Epidemiology of communityacquired pneumonia in adults: a population-based study. Eur Respir J 2000; 15:757-63.

4. Campbell JD, Sow SO, Levine MM, Kotloff KL. The causes of hospital admission and death among children in Bamako, Mali. J Trop Pediatr 2004 Jun; 503.-:158-63.

5. Dembek ZF, Carley K, Siniscalchi A, Hadler J. Hospital admissions syndromic surveillance-Connecticut, September 2001-November 2003. MMWR Morb Mortal Wkly Rep 2004 Sep 24; 53 Suppl:50-2.

6. Hviid A, Wohlfahrt J, Stellfeld M, Melbye M. Childhood vaccination and nontargeted infectious disease hospitalization. JAMA 2005 Aug 10; 294: 699-705.

7. Martin GS, Mannino DM, Eaton S, Moss M. The epidemiology of sepsis in the United States from 1979 through 2000. N Engl J Med 2003; 348: 1546-54. 
8. Koutsavlis AT. Hospital discharge databases in the surveillance of infectious diseases: Is their use warrented? Int J Infect Dis 2001; 5:59-62.

9. Baum S. Trends in ID-Related Hospitalizations -The Bugs Are Winning. J1 Watch Infect Dis 1988; 158:1813-8.

10. La Ribera Departamento 11 de Salud. Datos Demográficos en Area de Salud. Información Institucional http://www hospital-ribera com/, última visita 2007 Jul 22.

11. Mills CF, Tobias M, Baker M. A re-appraisal of the burden of infectious disease in New Zealand: aggregate estimates of morbidity and mortality. $\mathrm{N}$ Z Med J 2002; 115.

12. Jin S, Kilgore PE, Holman RC, Clarke MJ, Gangarosa EJ, Glass RI. Trends in hospitalizations for diarrhea in United States children from 1979 through 1992: estimates of the morbidity associated with rotavirus. Pediatr Infect Dis J 1996; 155: 397-404.

13. Mounts AW, Holman RC, Clarke MJ, Bresee JS, Glass RI. Trends in hospitalizations associated with gastroenteritis among adults in the United States, 1979-1995. Epidemiol Infect 1999; 1231.-:1-8.

14. Nelson EA, Tam JS, Yu LM, Glass RI, Parashar UD, Fok TF. Surveillance of childhood diarrhoeal disease in Hong Kong, using standardized hospital discharge data. Epidemiol Infect 2004; 4:619-26.

15. Gil A, Carrasco P, Jimenez R, San-Martin M, Oyaguez I, Gonzalez A. Burden of hospitalizations attributable to rotavirus infection in children in Spain, period 1999-2000. Vaccine 2004; 22:2221-5.

16. Visser LE, Cano Portero R, Gay NJ, Martinez Navarro JF. .Impact of rotavirus disease in Spain: an estimate of hospital admissions due to rotavirus. Acta Paediatr Suppl 1999; 88:72-6.

17. Davies GM, Dasbach EJ, Teutsch S. The burden of appendicitis-related hospitalizations in the United States in 1997. Surg Infect Larchmt.2004: 52:160-5.

18. Luckmann R. Incidence and case fatality rates for acute appendicitis in California. A population-based study of the effects of age. Am J Epidemiol 1989; 1295:905-18.
19. Telenti A. Estudio epidemiológico multicéntrico sobre neumonía en España. Med Clin (Barc). 1983; suppl 1:11-26.

20. Monge V, San Martin VM, Gonzalez A. The burden of community-acquired pneumonia in Spain. Eur J Public Health 2001; 4:362-4.

21. May DS, Kelly JJ, Mendlein JM, et al. Surveillance of major causes of hospitalization among the elderly, 1988. MMWR Morb Mortal Wkly Rep 1991; 40:7-21.

22. Pacheco A, González-Sainz J, Arocena C, Rebollar M, Antela A, Guerrero A. Communityacquired pneumonia caused by Chlamydia pneumoniae strain TWAR in chronic cardiopulmonary disease in the elderly. Respiration 1991; 58:31620.

23. Guerrero A, Wang SP, González Sáinz, Quereda C, Grayston JT. Prevalencia de la infección por Chlamydia pneumoniae en neumonías en una muestra de población hospitalaria española. Med Clin 1991; 96:285-6.

24. Guerrero A, González-Sainz J, Antela A. Epidemiología de la infección por Chlamydia pneumoniae en España. Enf Infec Microbiol Clin 1991; 910: 660-1.

25. Antela A, Guerrero A, Meseguer MA, GonzalezSainz U, Escudero R, Perez-Elias MJ, et al. Neumonías extrahospitalarias: estudio prospectivo de 101 pacientes adultos e inmunocompetente durante un año. Enferm Infecc Microbiol Clin 1993; 10:525-30.

26. Erice A, Romero-Vivas J, Martínez-Beltrán J, Guerrero A, Bouza E. Neumonía por Pseudomonas aeruginosa de adquisición extrahospitalaria en un paciente no inmunodeprimido. Med Clin (Barc). 1983; 81:811-2.

27. Leader S, Kohlhase K. Respiratory syncytial virus-coded pediatric hospitalizations, 1997 to 1999. Pediatr Infect Dis J 2002; 217:629-32.

28. Madsen KM, Schonheyder HC, Kristensen B, Nielsen GL, Sorensen HT. Can hospital discharge diagnosis be used for surveillance of bacteremia? A data quality study of a Danish hospital discharge registry. Infect Control Hosp Epidemiol 1998; 19:175-80. 